\title{
Role of $d$ electrons in electronic stopping of slow light ions
}

\author{
D. Goebl, ${ }^{*}$ D. Roth, and P. Bauer \\ Institut für Experimentalphysik, Abteilung für Atom- und Oberflächenphysik, Johannes Kepler University Linz, Altenberger Straße 69, \\ 4040 Linz, Austria
}

(Received 9 April 2013; published 27 June 2013)

\begin{abstract}
In recent energy loss measurements, band structure effects in electronic stopping have been observed for materials with finite excitation thresholds, for example, noble metals such as $\mathrm{Cu}$ and $\mathrm{Au}$. To further investigate the influence of the position of the $d$ band relative to the Fermi edge, electronic stopping of hydrogen and helium ions in $\mathrm{Ag}$ and $\mathrm{Pt}$ was determined. For $\mathrm{Ag}$, the electronic stopping power exhibits a velocity dependence similar to $\mathrm{Cu}$ and $\mathrm{Au}$. No particular effect due to the comparatively large $d$-band offset in Ag is found. In the case of $\mathrm{Pt}$, the electronic stopping power is virtually velocity proportional for $\mathrm{H}^{+}$ions and exhibits a distinct velocity dependence for $\mathrm{He}^{+}$ions. For hydrogen the results are compatible with modeling the conduction band as a free electron gas with an energy-dependent effective number of electrons. For $\mathrm{He}^{+}$, however, the observed effects point towards an additional energy loss mechanism, e.g., by charge-exchange processes.
\end{abstract}

DOI: 10.1103/PhysRevA.87.062903

PACS number(s): $34.50 . \mathrm{Bw}, 34.50 . \mathrm{Fa}$

\section{INTRODUCTION}

When an ion traverses matter it loses kinetic energy along its path, according to the stopping power $S=d E / d x$. These energy losses can be attributed to two different channels: elastic collisions with target nuclei and inelastic losses due to electronic excitations, the former being known as nuclear stopping power $S_{n}$, while the latter refers to the electronic stopping power $S_{e}$. To get rid of the trivial dependence of $S_{e}$ on the number density $n$, the electronic stopping cross section $\varepsilon$ is introduced which is defined as follows:

$$
\varepsilon=\frac{1}{n} S_{e}
$$

Since the early days of ion physics, a substantial amount of work has been devoted to the investigation of electronic stopping, both on a theoretical and experimental basis. Special emphasis was put on energy loss of light ions such as hydrogen or helium, as they present ideal model systems to study the underlying physical concepts. While for the electronic stopping power of light ions at medium to high energies the mechanisms are very well understood [1,2], the physical situation at low ion velocities-i.e., at ion velocities $v$ that are small compared to the Fermi velocity $v_{F}$-is still a matter of discussion. This regime is interesting insofar as the projectiles can only excite weakly bound electrons in the valence and conduction band of the target system. When the valence or conduction electrons are modeled as a free electron gas (FEG), the stopping power is known to exhibit velocity proportionality [3-5]:

$$
S_{e}=Q\left(Z_{1}, r_{s}\right) v .
$$

The proportionality factor $Q$, also denoted as friction coefficient, is a function of the atomic number of the projectile $Z_{1}$,

\footnotetext{
*dominik.goebl@jku.at

Published by the American Physical Society under the terms of the Creative Commons Attribution 3.0 License. Further distribution of this work must maintain attribution to the author(s) and the published article's title, journal citation, and DOI.
}

and the density parameter of the electron gas $r_{s}=(3 / 4 \pi n)^{1 / 3}$. At velocities $v<v_{F}$, velocity proportional stopping has indeed been observed for numerous target projectile combinations $[6,7]$.

In systems with finite excitation thresholds such as noble metals or insulators, recent investigations have shown that the stopping power for slow light ions exhibits a pronounced deviation from velocity proportionality [8-12]. The present study is concerned with a detailed investigation of the influence of $d$ electrons on electronic stopping. To this end, two different materials with distinct properties in the $d$-band configuration are chosen: $\mathrm{Ag}$ and $\mathrm{Pt}$ [13]. For $\mathrm{Ag}$, the $d$ band has a width of $\sim 4 \mathrm{eV}$ and extends up to $\sim 4 \mathrm{eV}$ below $E_{F}$. On the other hand, Pt exhibits a $\sim 8 \mathrm{eV}$ wide $d$ band which represents the dominant contribution to the electronic density of states at the Fermi energy.

In the present experiment, the stopping power is determined for atomic and molecular hydrogen and deuterium ions as well as for $\mathrm{He}^{+}$projectiles. For hydrogen one would not expect contributions to the stopping power due to projectile excitations - in contrast to $\mathrm{He}$, where it was shown recently that charge-exchange processes can contribute substantially to the electronic stopping power [14].

\section{EXPERIMENT AND EVALUATION}

Experiments were performed in the time-of-flight lowenergy ion scattering (TOF-LEIS) setup ACOLISSA [15]. As samples, evaporated films in the case of Ag and Au and a high purity (4N) sheet metal in the case of Pt were used. All samples were sputter-cleaned prior to measurement and surface cleanness was checked by Auger electron spectroscopy.

Electronic stopping powers of $\mathrm{Ag}$ and $\mathrm{Pt}$ were determined relative to a reference sample $(\mathrm{Au})$. The evaluation of the experiments was based on the fact that-within the single scattering model-for a given sample the spectrum height is inversely proportional to its stopping power. Contrary to previously used methods, which deduce the stopping power from a direct comparison of the experimental spectrum heights [12], we make use of Monte Carlo simulations to account for multiple scattering and nuclear stopping contributions 
[16]. The detailed evaluation procedure is described in the following for the case of Ag. Spectra were recorded for a material with known stopping power $(\mathrm{Au})$ and for the material under investigation $(\mathrm{Ag})$. From the experimental spectra the ratio of the heights $H_{\mathrm{Au}}^{\text {expt }} / H_{\mathrm{Ag}}^{\text {expt }}$ was determined. In addition, TRIM for Rutherford backscattering spectroscopy (TRBS) [17] simulations employing a Thomas-Fermi-Molière (TFM) potential [18] with a modified Firsov [19] screening length according to O'Connor and Biersack [20] were performed. TRBS allows for correction of its incorporated electronic stopping power values via a correction factor $c_{e}$. In the simulations the stopping power for $\mathrm{Au}$ was adapted to fit the experimental values [12] and the stopping power for Ag was initially left unchanged. From the resulting spectra a second height ratio, $H_{\mathrm{Au}}^{\mathrm{TRBS}} / H_{\mathrm{Ag}}^{\mathrm{TRBS}}$, was deduced. From these ratios, the electronic stopping power for $\mathrm{Ag}$ was then calculated using

$$
\varepsilon_{\mathrm{Ag}}^{\text {expt }}=\frac{H_{\mathrm{Au}}^{\text {expt }} / H_{\mathrm{Ag}}^{\text {expt }}}{H_{\mathrm{Au}}^{\mathrm{TRBS}} / H_{\mathrm{Ag}}^{\mathrm{TRBS}}} \frac{\varepsilon_{\mathrm{Au}}^{\text {expt }}}{\varepsilon_{\mathrm{Au}}^{\mathrm{TRBS}}} \varepsilon_{\mathrm{Ag}}^{\mathrm{TRBS}} .
$$

This evaluation was applied for a certain energy interval slightly below the high energy edge $\left(k_{\mathrm{Ag}} E_{0}\right.$, with the kinematic factor $k$ ) of the silver spectrum. With the resulting $\varepsilon_{\mathrm{Ag}}^{\text {exp }}$, TRBS simulations were repeated and their results were evaluated again using Eq. (3). This procedure was iterated until convergence in $\varepsilon_{\mathrm{Ag}}^{\text {expt }}$ was reached. Figure 1 illustrates this for $6 \mathrm{keV} \mathrm{H}^{+}$ions scattered from $\mathrm{Ag}$ and $\mathrm{Au}$, respectively. Figure 1(a) displays the experimental and simulated spectra with the final stopping power $\varepsilon_{\mathrm{Ag}}^{\text {expt }}$. Figure 1(b) displays the corresponding experimental and simulated height ratios as a function of the energy. The vertical lines in both parts of Fig. 1 indicate the region in which the height ratios are evaluated.

We consider this method to be accurate within $\sim 5 \%-15 \%$ standard deviation for each obtained value, depending on ion velocity. In the following we summarize a number of factors, which should be considered in the evaluation, and their possible influence on the results:

(a) Beam current stability: In the evaluation the ion current is assumed to be constant. Thus, it is essential to ensure a stable primary beam current during the measurements. To this end, the measurement at a certain incident energy is split into three parts: measurement of sample 1 with acquisition time $T / 2$, measurement of sample 2 with acquisition time $T$, and a second measurement of sample 1 with acquisition time $T / 2$. From comparison of the first and last spectra, one can easily identify instabilities of the primary beam current. Since in our TOF experiment the beam currents are too small to be measured precisely $(\sim 1-10 \mathrm{pA})$, the multichannel analyzer (MCA) signal was recorded in list mode and converted to a counts-per-second format. From this, one always could see whether the current was sufficiently stable to make an evaluation meaningful; in some cases it permitted us to correct for changes in the beam current. Small fluctuations of the beam current are expected to contribute to the statistical uncertainty of the results.

(b) Sample cleanness: surface contaminations such as a monolayer of water or carbon would shift spectra by $\sim 20 \mathrm{eV}$ at $v=0.6$ a.u. $\left(9 \mathrm{keV} \mathrm{H}^{+}\right)$and by $\sim 11 \mathrm{eV}$ at $v=0.14$ a.u. $\left(1 \mathrm{keV} \mathrm{D}^{+}\right)$. For high energies, this shift is small compared

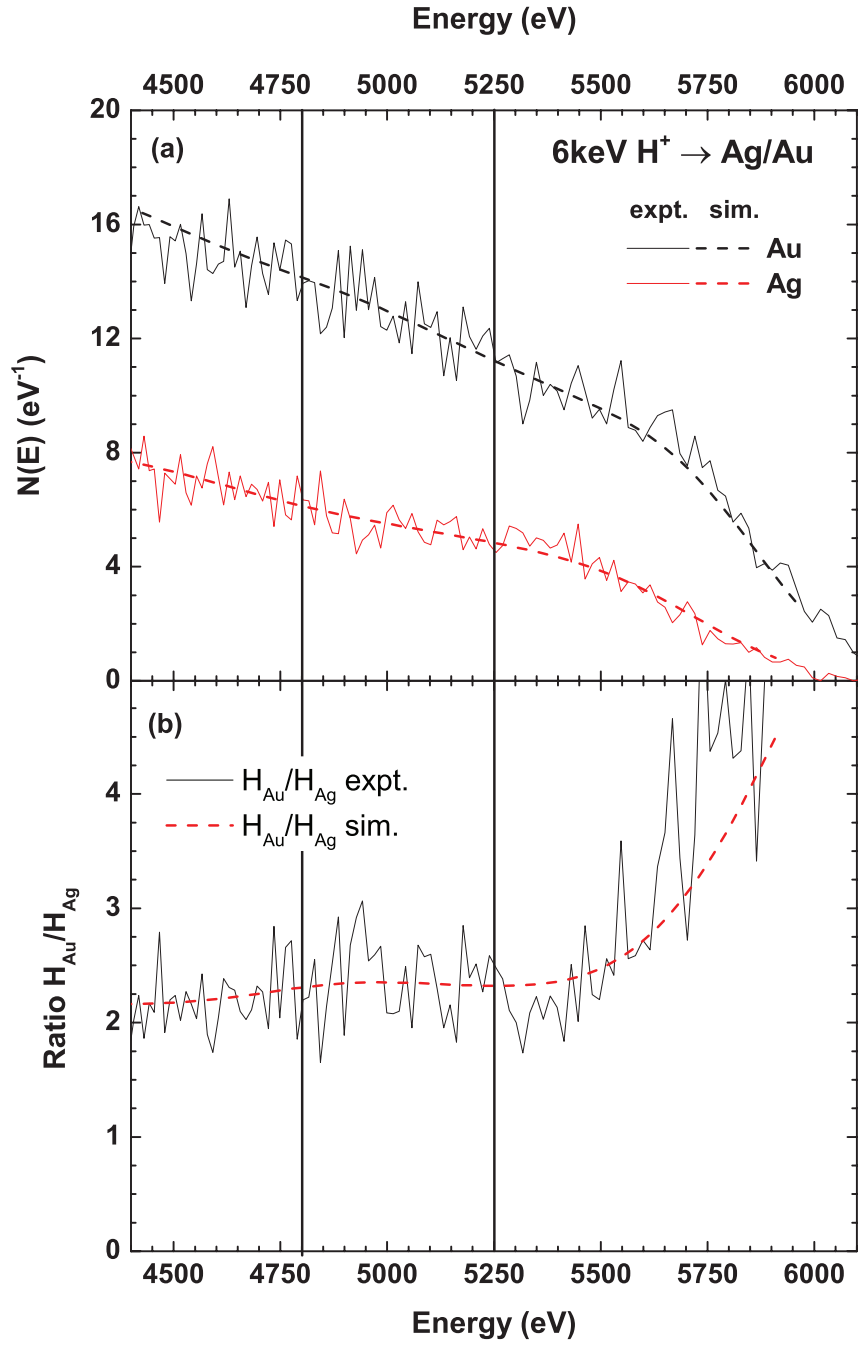

FIG. 1. (Color online) (a) Experimental (solid lines) and TRBSsimulated (dashed lines) spectra of $6 \mathrm{keV} \mathrm{H}^{+}$scattered from thick $\mathrm{Au}$ (black) and Ag [red (gray)] targets. Stopping power in the simulation of $\mathrm{Au}$ spectra was chosen from Ref. [12]; stopping power in the simulation of $\mathrm{Ag}$ was corrected to fit experimental data. Horizontal lines mark the region which is analyzed to perform corrections to the Ag stopping power. (b) Experimental and simulated height ratios as a function of energy. Both height ratios are integrated over the indicated energy range (4800-5250 eV) and the resulting values are taken to evaluate the ratio of the height ratios.

to the energy window and thus the effect on the resulting stopping cross section should be negligible. For low energies, however, this shift is comparable to the width of the evaluation interval. Nevertheless, even when one considers shifts of this magnitude in the evaluation (by shifting both or just one of the experimental spectra), influences on the final $\varepsilon$ values are typically $<10 \%$. Consequently, surface contaminations may represent a possible source of a systematic error in the order $< \pm 10 \%$.

(c) Evaluation: To minimize the influence of multiple scattering or nuclear stopping-especially at low energiesthe evaluation interval should start just below $k E_{0}$ and the width should be limited, in order to facilitate convergence. In this study, the interval was selected such that its width was smaller than $10 \%$ of the primary beam energy. Note 


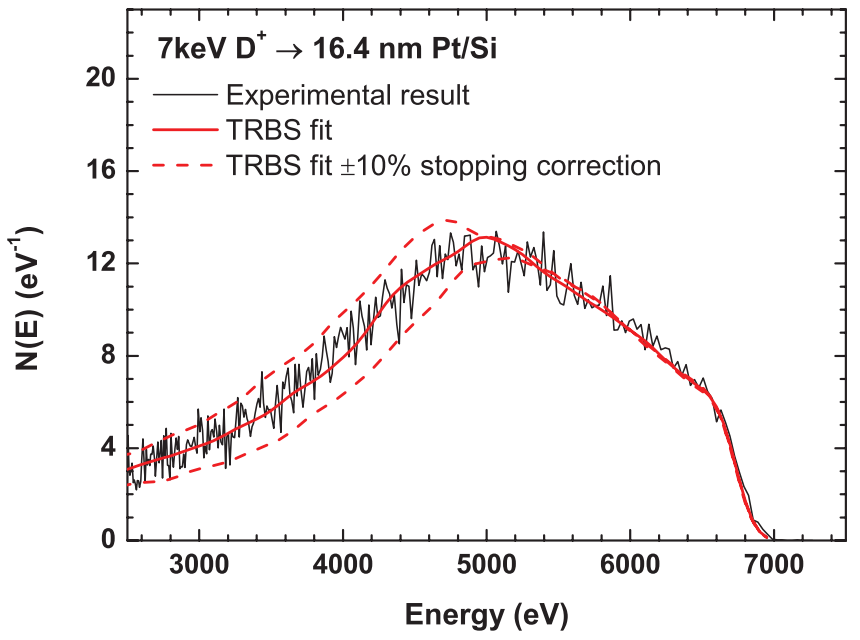

FIG. 2. (Color online) Energy spectrum of $7 \mathrm{keV} \mathrm{D}^{+}$scattered from a thin $(16.4 \mathrm{~nm}) \mathrm{Pt}$ film. Black line corresponds to the experimental values; red line represents simulated data (TRBS). The stopping power value was determined by choosing the appropriate stopping correction factor in TRBS. To illustrate the sensitivity of this method, simulated results with a modified stopping $( \pm 10 \%)$ are shown (red dashed line).

that especially at lower energies it becomes delicate to fix the position of the evaluation interval. We consider this fact together with beam current instabilities to be the main reason for the higher statistical uncertainty at the lowest velocities. Of course the uncertainty in the stopping power of the reference material also leads to a corresponding systematic uncertainty of the resulting stopping power.

To confirm the results obtained in relative measurements, spectra were recorded for thin evaporated $\mathrm{Ag}$ and $\mathrm{Pt}$ films. In the case of Ag a thickness of $21.1 \pm 1.1 \mathrm{~nm}$ was determined by Rutherford backscattering spectroscopy (RBS) measurements with $500 \mathrm{keV}$ protons relative to a Au sample of known thickness. The deduced thickness was confirmed by $2 \mathrm{MeV}$ $\mathrm{He}^{+} \mathrm{RBS}$ measurements at Uppsala University. The $\mathrm{Pt}$ sample with a thickness of $16.4 \mathrm{~nm}$ was provided by Daniel Primetzhofer, Uppsala University. From the widths of these spectra it is possible to deduce the stopping power by a direct comparison to TRBS simulations (see Fig. 2). As already mentioned, TRBS allows for a correction of its incorporated stopping power by a single correction factor. If the stopping power used in TRBS is not proportional to the experimental stopping power, a systematic error is introduced in the evaluated stopping power, especially when a wide velocity range is probed. To minimize this error, the stopping power is evaluated at a mean energy $\langle E\rangle=\left(E_{0}+E_{\min }\right) / 2$, where $E_{\text {min }}$ corresponds to the low-energy edge of the thin film spectrum.

\section{RESULTS AND DISCUSSION}

The electronic stopping cross section of hydrogen in Ag is depicted in Fig. 3. Figures 3(a) and 3(b) display identical data in different formats: (a) stopping cross section $\varepsilon$ as a function of ion velocity and (b) friction coefficient $\varepsilon / v$ as a function of ion velocity. Results obtained with $\mathrm{H}^{+}, \mathrm{H}_{2}{ }^{+}$, and $\mathrm{H}_{3}{ }^{+}$are shown as squares, while data from $\mathrm{D}^{+}, \mathrm{D}_{2}{ }^{+}$, and $\mathrm{D}_{3}{ }^{+}$are represented

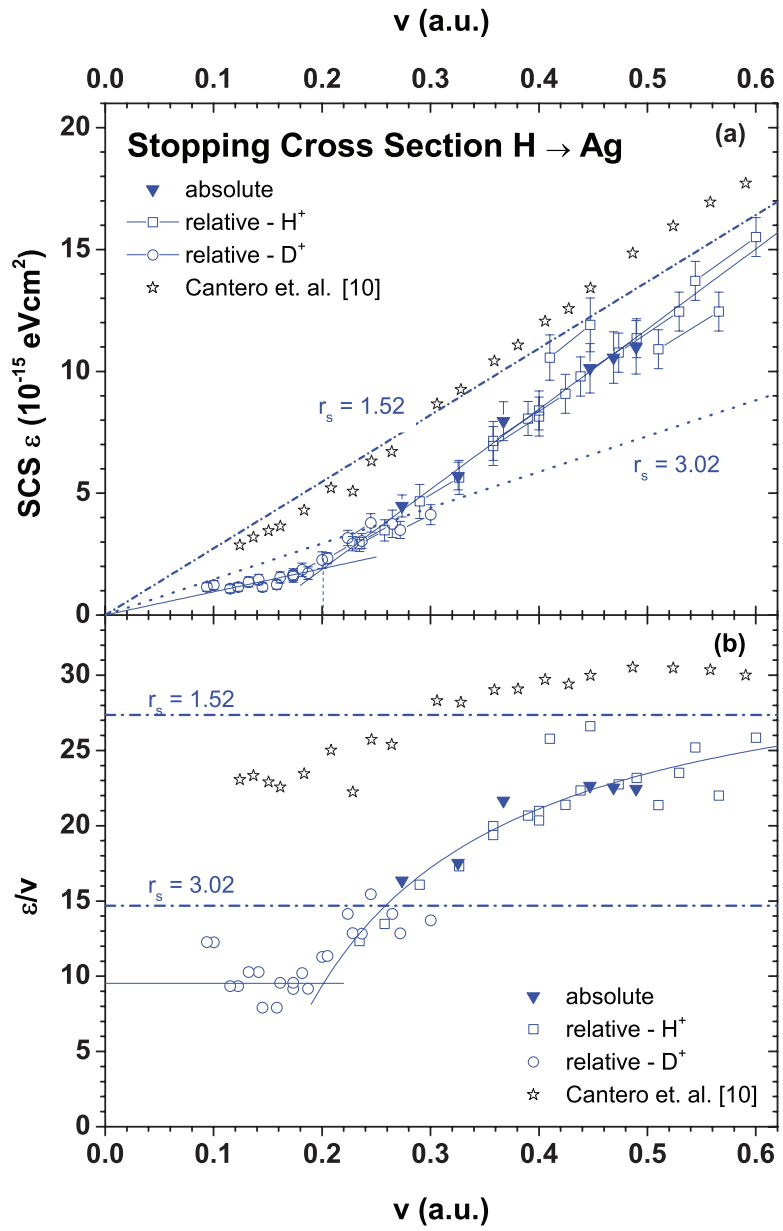

FIG. 3. (Color online) (a) Stopping cross section for elemental and molecular hydrogen (blue squares) and deuterium (blue circles) ions in $\mathrm{Ag}$ as a function of ion velocity. Open symbols correspond to relative measurement to $\mathrm{Au}$, full symbols denote absolute measurements of a thin Ag film. Black asterisks represent previously measured data [11]. Dashed and dash-dotted line indicate results of DFT calculations for different values of $r_{s}$ [5]. (b) Friction coefficient $\varepsilon / v$ for the data presented in (a) as a function of ion velocity.

by circles. Both types of ions lead to concordant results, and no difference between data from atomic and molecular ions is observed. This indicates negligible influence of vicinage or isotope effects. There is excellent agreement between data obtained in relative and absolute measurements. Since absolute and relative measurements make use of completely different types of information, they may be influenced by completely different systematic errors. Therefore, the high level of agreement between the results from these two types of measurements gives very much confidence in the results and systematic errors are certainly below the statistical uncertainties. A chi-squared test of the experimental data to the depicted linear fits (continuous lines) reveals a mean statistical uncertainty of $14 \%$ for velocities $<0.2$ a.u. and a mean error of $10 \%$ for $v>0.2$ a.u.

The stopping power of $\mathrm{Ag}$ for $\mathrm{H}$ exhibits the behavior well known from the other noble metals, $\mathrm{Cu}$ and $\mathrm{Au}[9,11,12]$ : At low velocities, i.e., $v<\sim 0.2$ a.u., stopping is mostly due to the interaction of the projectile with the $5 \mathrm{~s}$ electrons 
and the stopping cross section is proportional to velocity. At $v \approx 0.2$ a.u. a pronounced deviation from velocity proportionality is observed due to increasing contributions from $d$ electrons to the stopping power. Additionally, friction coefficients calculated by density functional theory (DFT) [5] for $r_{s}$ values of 3.02 and 1.52 are included in Fig. 3 (dash-dotted lines). The $r_{s}$ value of 3.02 corresponds to a FEG that mimics a single Ag $5 s$ electron, while $r_{s}=1.52$ is deduced from experimentally obtained plasmon energies [21] and corresponds to the interaction of the ion with all conduction electrons. For low velocities $(<0.2$ a.u.), the experimental values are slightly below the theoretical predictions while for higher energies experimental values nicely approach the theoretical value.

When the presented data are compared to the data obtained by Cantero et al. [11] one can see a striking difference in the absolute values. Although the kink velocity is reproduced very well, the data from Ref. [11] are higher than our data at low projectile velocities, by up to a factor of 2.5 . This large discrepancy decreases at higher velocities: At a velocity of 0.6 a.u. the difference is only $20 \%$. The robustness of our evaluation method together with the fact that we obtain concordant results for two different evaluation methods (absolute and relative) give confidence in our results. One obvious difference to our experiment is that in Ref. [11] the data were obtained in transmission geometry. In order to find the source for the observed differences a thorough investigation will be required. The optimum would be to use one set of samples which is analyzed in both setups.

The electronic stopping cross section for $\mathrm{He}^{+}$ions in $\mathrm{Ag}$ is shown in Fig. 4. The results are presented in the same fashion as for $\mathrm{H}$ in Fig. 3. Measurements were performed relative to a $\mathrm{Au}$ sample (open squares) as well as for a thin Ag film (full squares). Relative and absolute measurements are in good agreement within the given statistical uncertainty of $\sim 10 \%$. When $\mathrm{He}$ is used as a projectile, complications may arise due to the difference in kinematic factors $k_{\mathrm{Ag}}=0.886$ and $k_{\mathrm{Au}}=$ 0.936 . The experimental and simulated ratios are compared for energies below $k_{\mathrm{Ag}} E_{0}$, which neglects the Au spectrum in the interval $\left[k_{\mathrm{Ag}} E_{0}, k_{\mathrm{Au}} E_{0}\right]$. Considering the evaluation procedure, this fact should not have a significant influence on the result as long as the stopping cross section exhibits velocity proportionality. However, to test the robustness of the method a modified evaluation procedure was performed: Before the ratios are calculated, the simulated and experimentally obtained Ag spectra were shifted in energy until the high energy edges coincided with those of Au. Apart from statistical variations $<10 \%$, the resulting values for $\varepsilon$ did not show any deviation from the values obtained with the standard method.

In the experimental data, one can see a clear change in the velocity dependence at an ion velocity of $\sim 0.185$ a.u. Fits of the data in the different regimes (straight lines) are given to guide the eye of the reader. Figure 4 also contains $r_{s}$ dependent friction coefficients calculated by DFT [5] as in Fig. 3. Theoretical predictions underestimate $\left(r_{s}=3.02\right)$ or overestimate $\left(r_{s}=1.52\right)$ the experimental data in the same way as was observed for $\mathrm{Au}$ [12] and may be related to projectile excitation [22].

Figure 5 combines the present results for $\mathrm{Ag}$ and stopping data for $\mathrm{Au}$ and $\mathrm{Cu}$ [12], to obtain a complete picture

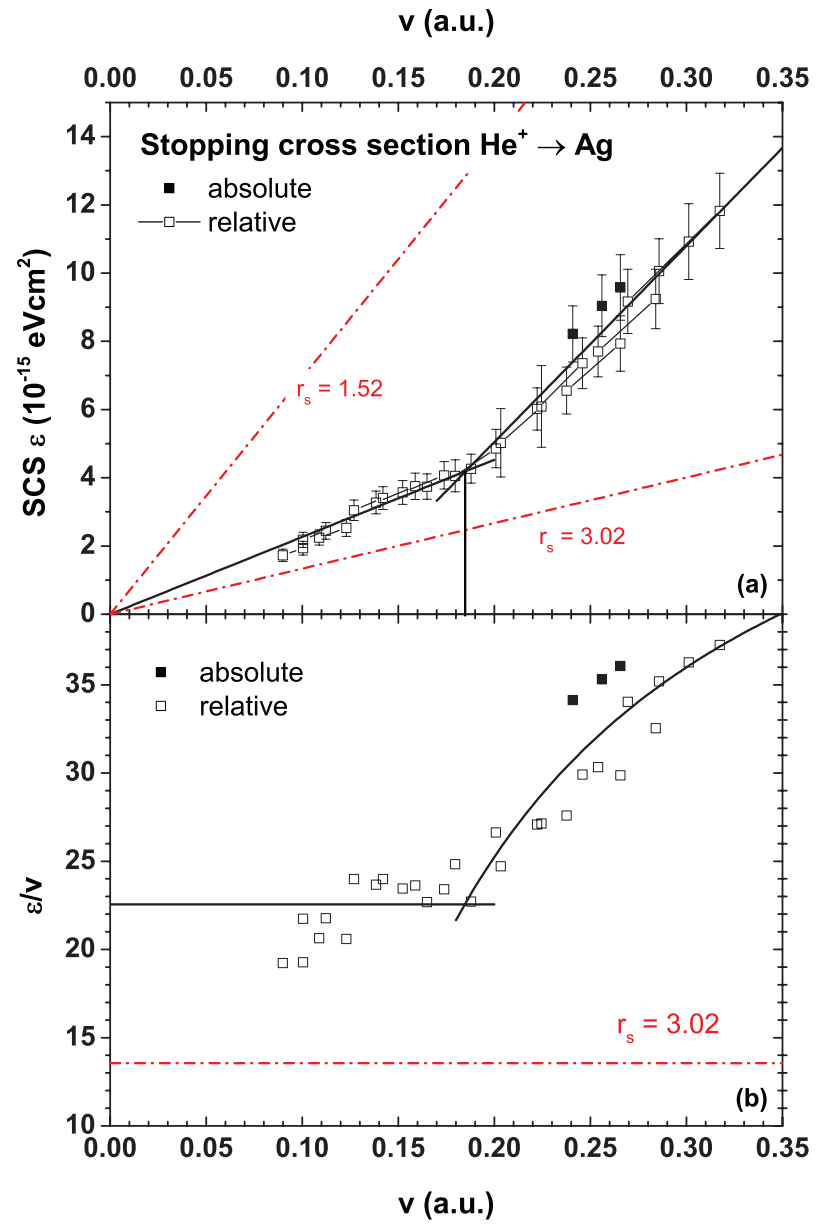

FIG. 4. (Color online) (a) Stopping cross section for $\mathrm{He}^{+}$ions in $\mathrm{Ag}$ as a function of ion velocity. Relative measurements (open squares) and absolute measurements (full squares) are shown together with theoretical predictions based on DFT [5] for different electron densities (dash-dotted lines). (b) Friction coefficient $\varepsilon / v$ for the data presented in (a) as a function of ion velocity.

of $\mathrm{H}^{+}$[Fig. 5(a)] and $\mathrm{He}^{+}$[Fig. 5(b)] stopping in noble metals. Both figures contain theoretical predictions according to DFT calculations (dotted lines) with electron densities corresponding to a FEG with one electron $\left(\mathrm{Cu}: r_{s}=2.67\right.$ a.u., Ag: $r_{s}=3.02$ a.u., Au: $r_{s}=3.01$ a.u.).

For the different projectiles, the following observations can be made:

$\mathrm{H}^{+}, \mathrm{He}^{+}$. In the low energy region ( $v<0.2$ a.u.), all noble metals exhibit velocity proportional electronic stopping cross sections.

$\mathrm{H}^{+}$. The stopping power of $\mathrm{Ag}$ is lower compared to $\mathrm{Au}$, by $15 \%$, in qualitative agreement with a prediction based on more general arguments [23]. Deviation from velocity proportionality starts at $\sim 0.175$ a.u. for $\mathrm{Cu}, \sim 0.19$ a.u. for $\mathrm{Au}$, and $\sim 0.20$ a.u. for $\mathrm{Ag}$. The overall agreement with theory is good, with discrepancies up to $\sim 50 \%$.

$\mathrm{He}^{+}$. Deviation from velocity proportionality starts at $\sim 0.19$ a.u. for $\mathrm{Cu}, \mathrm{Ag}$, and $\mathrm{Au}$. Experimental data exceed theoretical predictions by theory for all three materials by up to $\sim 120 \%$. 


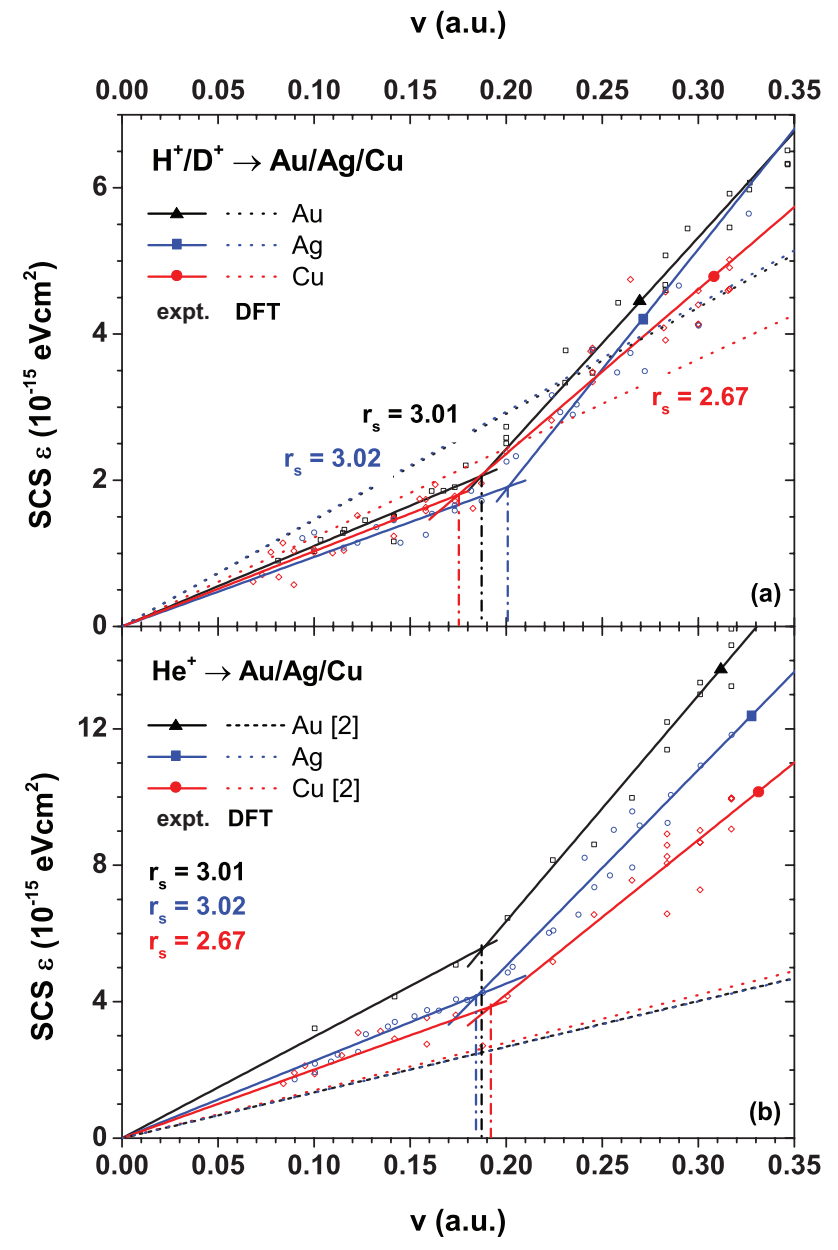

FIG. 5. (Color online) Electronic stopping cross section of the noble metals for hydrogen (a) and helium (b) ions. Ag data obtained in this investigation is compared to previously measured data [12].

Special attention should be directed towards the observed kink velocities $v_{k}$, i.e., the velocity where one can observe a deviation from velocity proportionality. One possible interpretation is that $v_{k}$ is proportional to the excitation threshold of the system [12]. In the case of the noble metals the excitation threshold is given by the $d$-band offset in the system $(\mathrm{Au}=$ $2 \mathrm{eV}, \mathrm{Cu}=2 \mathrm{eV}, \mathrm{Ag}=4 \mathrm{eV}$ ) [13,24]. Thus, one would expect that $\mathrm{Ag}$ exhibits a significantly higher $v_{k}$ than $\mathrm{Au}$ or $\mathrm{Cu}$. This expectation is - at least on a qualitative basis - partly fulfilled for $\mathrm{H}^{+}$ions. For $\mathrm{He}^{+}$ions, in contrast, the kink velocities of $\mathrm{Cu}, \mathrm{Ag}$, and $\mathrm{Au}$ virtually coincide. However, one should bear in mind that the determined kink velocities are only a qualitative quantity. In a more realistic description, Zeb and co-workers have shown that excitation of $d$ electrons starts already at rather low projectile velocities, and strongly increases with velocity [25].

Compared to $\mathrm{Ag}$, the electronic stopping power of $\mathrm{Pt}$ for hydrogen shows a qualitatively different picture as can be seen in Fig. 6(a). Here, the stopping power of $\mathrm{H}$ (squares) and D (circles), obtained in relative measurements (open symbols) and absolute measurements (full symbols) is shown. Similar to $\mathrm{Ag}$, different isotopes as well as molecular ions exhibit consistent stopping power data within statistical uncertainty. Experimental data show almost no deviation from velocity

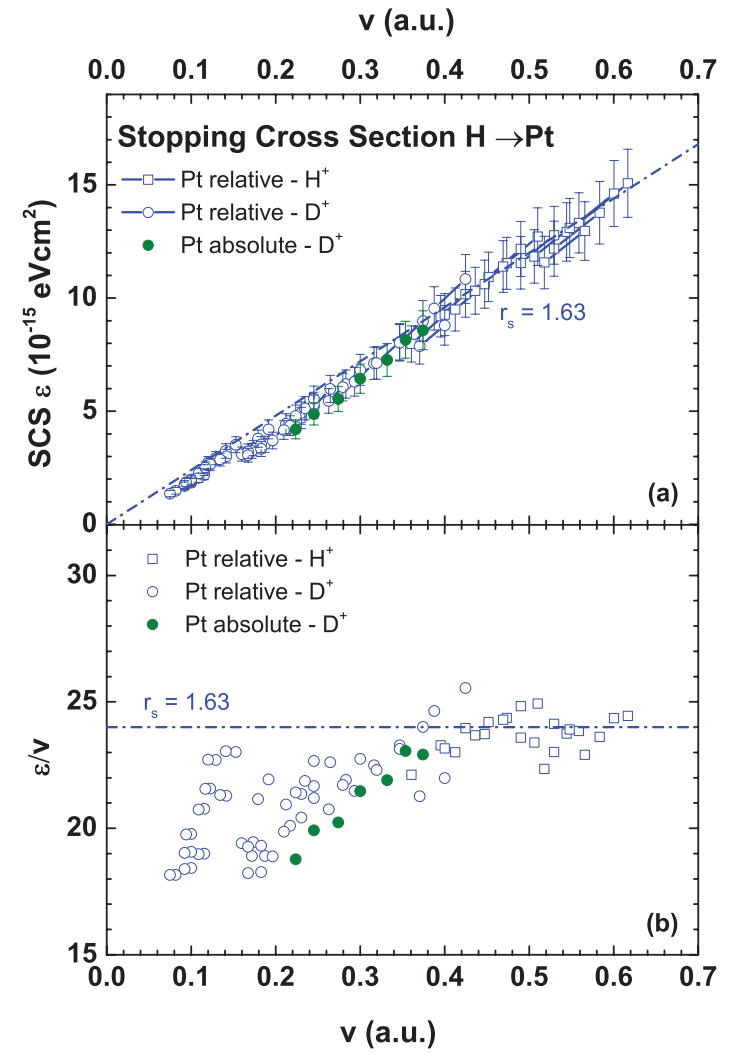

FIG. 6. (Color online) (a) Stopping cross section for elemental and molecular hydrogen (blue squares) and deuterium (blue circles) ions in $\mathrm{Pt}$ as a function of ion velocity. Open symbols correspond to relative measurement to $\mathrm{Au}$; full symbols denote absolute measurements of a thin Pt film. Dash-dotted line represents results of DFT calculations [5]. (b) Friction coefficient $\varepsilon / v$ for the data presented in (a) as a function of ion velocity.

proportional stopping over the covered velocity range, and also agree very well with previously measured data in the energy range between 30 and $300 \mathrm{keV}$ [22]. However, the corresponding friction coefficient data, $\varepsilon / v$, [see Fig. 6(b)] exhibit a minor increase when going from lower to higher velocities. Similar to the noble metals, the average friction coefficient is lower for $v<\sim 0.2$ a.u. as compared to that at velocities $>0.2$ a.u. by about $20 \%$. This behavior may be based on different excitation efficiencies for $d$ and $s$ electrons [25]. One should, however, keep in mind that the magnitude of this effect is of the order of one standard deviation per data point. For linear fits to the two regions below and above $v=0.2$ a.u., chi-squared tests revealed statistical uncertainties of $8.5 \%$ and $3.5 \%$ in the lower and upper velocity regimes, respectively. The experimental data are compared to predictions by DFT calculations for a FEG, with $r_{s}=1.63$ (deduced from experimental plasmon energies [21]). The DFT results are in perfect agreement with our data.

The stopping power data for $\mathrm{He}^{+}$in $\mathrm{Pt}$ is presented in Fig. 7(a); the corresponding friction coefficients as a function of ion velocity are shown in Fig. 7(b). Data were acquired relative to $\mathrm{Au}$ (open symbols) and in absolute measurements using thin Pt films (full symbols). Theoretically predicted friction coefficients according to DFT calculations [5] are presented by dash-dotted lines; the same $r_{s}$ value as in 


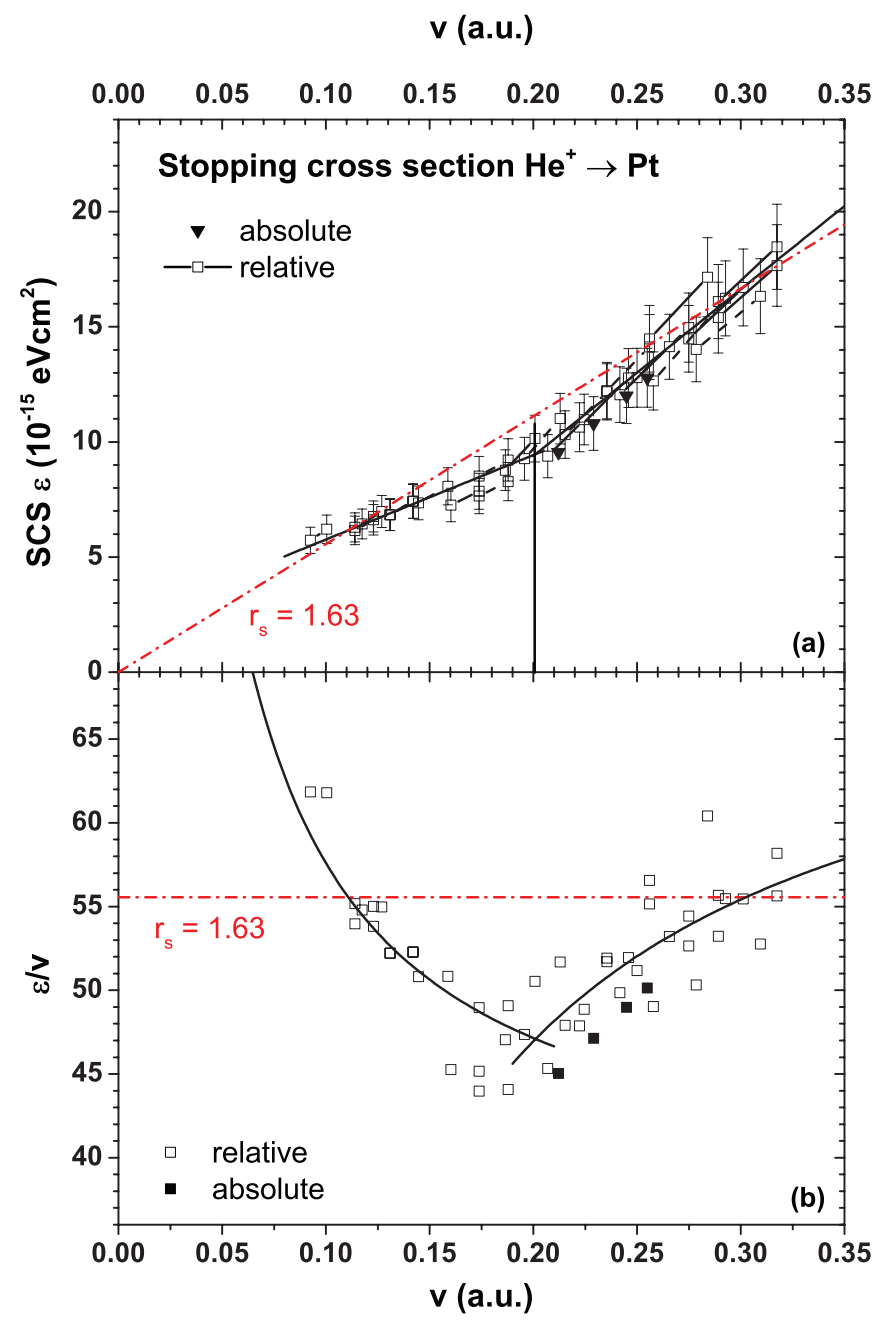

FIG. 7. (Color online) (a) Stopping cross section for $\mathrm{He}^{+}$ions in $\mathrm{Pt}$ as a function of ion velocity. Presented data were obtained in relative measurements (open squares) and absolute measurements (full squares). Theoretical prediction based on DFT [5] for an electron density, $r_{s}=1.63$, is included in the plot (dash-dotted line). (b) Friction coefficient $\varepsilon / v$ for the data presented in (a) as a function of ion velocity.

Fig. 6 is employed. Absolute and relative measurements are in excellent agreement and also agree very well to previously measured data around the stopping maximum [22]. Also for $\mathrm{He}^{+}$and $\mathrm{Pt}$, the experimental data exhibit a change in the velocity dependence at an ion velocity of $\sim 0.2$ a.u. The low-velocity data are well described by a linear fit pointing to a positive offset on the $y$ axis. Such a velocity dependence at very low velocities has never been observed before. On the basis that $\varepsilon$ has to vanish at $v=0$, the deviation from velocity proportionality at low velocities may be a result of charge-exchange processes between $\mathrm{Pt}$ and $\mathrm{He}^{+}$, with the efficiency of these processes having a nonlinear energy dependence. To support this interpretation, electrostatic analyzer (ESA)-LEIS measurements for $\mathrm{He}^{+}$scattered from a polycrystalline Pt surface were performed. The deduced ion fraction $P^{+}$, as a function of the inverse perpendicular velocity $v_{\perp}$, is shown Fig. 8 together with $P^{+}$for He and Ag [26]. As one can see from Fig. $8, P^{+}\left(1 / v_{\perp}\right)$ can be described by a

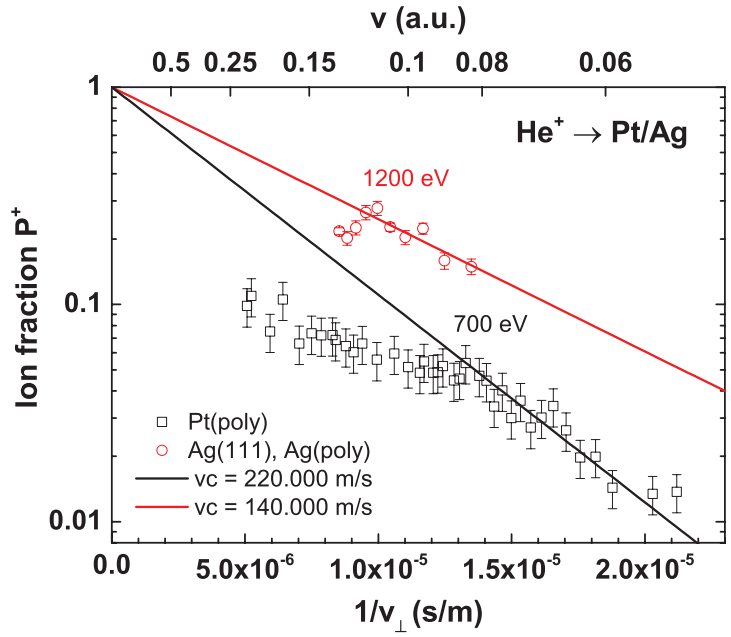

FIG. 8. (Color online) Ion fraction $P^{+}$for $\mathrm{He}^{+}$scattered from polycrystalline $\mathrm{Pt}$ (black squares) as well as from polycrystalline $\mathrm{Ag}$ and $\operatorname{Ag}(111)$ (red circles) [26]. Straight lines correspond to singleexponential fits of the data. The apparent threshold energy for resonant processes in a close collision is indicated for both materials. Note that the given velocity values (top $x$ axis) are only valid for Pt (the relation between $1 / v_{\perp}$ and velocity depends on the $k$ factor of the target material [27]).

single exponential, $\exp \left(-v_{c} / v_{\perp}\right)$, for sufficiently low primary energies, $E_{0}<E_{\mathrm{th}}$, with $E_{\mathrm{th}}$ the threshold energy for resonant charge-exchange processes in a close collision (neutralization and re-ionization) [27]. This behavior indicates that in this regime, neutralization is exclusively due to Auger neutralization (AN). The characteristic velocity $v_{c}$ is significantly higher for $\mathrm{Pt}(220.000 \mathrm{~m} / \mathrm{s})$ than for $\mathrm{Ag}(140.000 \mathrm{~m} / \mathrm{s})$; this can be understood as a band structure effect in Auger neutralization [28]. For $\mathrm{Pt}, E_{\text {th }}$ is considerably lower than for $\mathrm{Ag}$, i.e., $700 \mathrm{eV}$ for $\mathrm{Pt}$, compared to $1200 \mathrm{eV}$ for $\mathrm{Ag}$. As has been observed for other materials, also in the present case resonant neutralization in a close collision is more effective than re-ionization in the vicinity of $E_{\text {th }}$ [29]; this conclusion is based on the observation that at $E_{0}>E_{\mathrm{th}}$, $P^{+}$is lower than expected from pure AN. The fact that for $\mathrm{Pt}, P^{+}$increases at primary energies $E_{0}>1 \mathrm{keV}$ points to an increased relative importance of re-ionization. In contrast, previous measurements of $P^{+}$for $\mathrm{He}^{+}$scattered from $\mathrm{Ag}(110)$ do not show a significant increase of $P^{+}$in the corresponding energy range [30]. Since re-ionization in combination with AN has to be considered a possible energy loss mechanism [14], one might expect charge-exchange processes to contribute at lower energies for $\mathrm{He}-\mathrm{Pt}$ than for $\mathrm{He}-\mathrm{Ag}$. Furthermore, also at energies around the stopping maximum, charge-exchange has been proposed as a mechanism to explain deviations from velocity proportionality in the He/Pt system [22].

\section{CONCLUSION}

We have presented experimentally obtained electronic stopping cross section data of $\mathrm{Ag}$ and Pt for slow hydrogen, deuterium, and helium ions. Ag exhibits a similar behavior as $\mathrm{Au}$ and $\mathrm{Cu}$ : velocity proportional stopping at low projectile velocities followed by a pronounced deviation at $\sim 0.2$ a.u. 
The experimental data confirm that there is no simple linear relationship between excitation threshold and kink velocity.

In the case of $\mathrm{H}^{+}$in $\mathrm{Pt}$, we have found only little deviation from velocity proportional stopping. These results once more confirm that $\mathrm{H}$ ions are ideal probes when one looks for band structure effects in electronic stopping, since for $\mathrm{H}$ ions projectile excitations do not contribute noticeably to the inelastic losses. In principle, this allows one to model the electronic stopping power of materials with complex band structures based on a FEG with an energy-dependent effective number of electrons. However, in the case of $d$ electrons one has to keep in mind that contrary to pure FEG metals such as $\mathrm{Al}$, the $d$ electrons may influence the results also for very small velocities due to screening effects [25]. The stopping power for $\mathrm{He}^{+}$in Pt exhibits a more complex behavior. The present data may point towards a process which increases energy loss at velocities $<0.1$ a.u.
Additionally, we presented an evaluation method to obtain stopping power data for a certain material based on the comparison of experimental and simulated spectra to a reference material. Results obtained by this method showed excellent agreement to results obtained by backscattering from a thin film, and thus, confirmed this method as a valid evaluation technique.

\section{ACKNOWLEDGMENTS}

We express our gratitude to Daniel Primetzhofer, who provided the thin Pt film samples and also performed the RBS measurements in Uppsala. Support by the Austrian Science Fund (FWF): Project P22587 is gratefully acknowledged. D.G. was supported by a DOC Fellowship of the Austrian Academy of Sciences.
[1] C. P. Race, D. R. Mason, M. W. Finnis, W. M. C. Foulkes, A. P. Horsfield, and A. P. Sutton, Rep. Prog. Phys. 73, 116501 (2010).

[2] P. Sigmund, Particle Penetration and Radiation Effects: General Aspects and Stopping of Swift Point Charges (Springer, Berlin, New York, 2008).

[3] J. Lindhard, K. Dan. Vidensk. Selsk., Mat.-Fys. Medd. 28, 8 (1954).

[4] P. M. Echenique, R. M. Nieminen, and R. H. Ritchie, Solid State Commun. 37, 779 (1981).

[5] I. Nagy, A. Arnau, and P. M. Echenique, Phys. Rev. A 40, 987 (1989).

[6] S. P. Møller, A. Csete, T. Ichioka, H. Knudsen, U. I. Uggerhøj, and H. H. Andersen, Phys. Rev. Lett. 93, 042502 (2004).

[7] H. Bitao, L. Zhaoyuan, and Q. Zhong, Nucl. Instrum. Methods Phys. Res., Sect. B 149, 395 (1999).

[8] G. Martínez-Tamayo, J. C. Eckardt, G. H. Lantschner, and N. R. Arista, Phys. Rev. A 54, 3131 (1996).

[9] E. A. Figueroa, E. D. Cantero, J. C. Eckardt, G. H. Lantschner, J. E. Valdés, and N. R. Arista, Phys. Rev. A 75, 010901(R) (2007).

[10] S. N. Markin, D. Primetzhofer, S. Prusa, M. Brunmayr, G. Kowarik, F. Aumayr, and P. Bauer, Phys. Rev. B 78, 195122 (2008).

[11] E. D. Cantero, G. H. Lantschner, J. C. Eckardt, and N. R. Arista, Phys. Rev. A 80, 032904 (2009).

[12] S. N. Markin, D. Primetzhofer, M. Spitz, and P. Bauer, Phys. Rev. B 80, 205105 (2009).

[13] N. Smith, G. Wertheim, S. Hüfner, and M. Traum, Phys. Rev. B 10, 3197 (1974).

[14] D. Primetzhofer, S. Rund, D. Roth, D. Goebl, and P. Bauer, Phys. Rev. Lett. 107, 163201 (2011).
[15] M. Draxler, S. N. Markin, S. N. Ermolov, K. Schmid, C. Hesch, A. Poschacher, R. Gruber, M. Bergmann, and P. Bauer, Vaccum 73, 39 (2004).

[16] D. Roth, D. Goebl, and P. Bauer, Nucl. Instrum. Methods Phys. Res., Sect. B (2013) doi:10.1016/j.nimb.2012.12.094.

[17] J. P. Biersack, E. Steinbauer, and P. Bauer, Nucl. Instrum. Methods Phys. Res., Sect. B 61, 77 (1991).

[18] G. Molière, Z. Naturforsch., A: Phys. Sci. 2, 133 (1947).

[19] O. B. Firsov, Sov. Phys. JETP 6, 534 (1958).

[20] D. O'Connor and J. P. Biersack, Nucl. Instrum. Methods Phys. Res., Sect. B 15, 14 (1986).

[21] H. Raether, Excitation of Plasmons and Interband Transitions by Electrons (Springer-Verlag, Berlin, New York, 1980).

[22] D. Primetzhofer, Phys. Rev. B 86, 094102 (2012).

[23] D. Semrad and R. Golser, Phys. Rev. A 35, 4836 (1987).

[24] J. Rowe and N. Smith, Phys. Rev. B 10, 3207 (1974).

[25] M. A. Zeb, J. Kohanoff, D. Sánchez-Portal, A. Arnau, J. I. Juaristi, and E. Artacho, Phys. Rev. Lett. 108, 225504 (2012).

[26] D. Primetzhofer, S. N. Markin, J. I. Juaristi, E. Taglauer, and P. Bauer, Nucl. Instrum. Methods Phys. Res., Sect. B 267, 624 (2009).

[27] H. Brongersma, M. Draxler, M. Deridder, and P. Bauer, Surf. Sci. Rep. 62, 63 (2007).

[28] D. Goebl, D. Valdés, E. Abad, R. C. Monreal, D. Primetzhofer, and P. Bauer, Phys. Rev. B 84, 165428 (2011).

[29] D. Primetzhofer, M. Spitz, E. Taglauer, and P. Bauer, Surf. Sci. 605, 1913 (2011).

[30] D. Primetzhofer, M. Spitz, S. N. Markin, E. Taglauer, and P. Bauer, Phys. Rev. B 80, 125425 (2009). 